\title{
A novel approach to flow quantification in brain arteriovenous malformations prior to enbucrilate embolization: use of insoluble contrast (Ethiodol droplet) angiography
}

\begin{abstract}
Ajay K. Wakhloo, M.D., Ph.D., Baruch B. Lieber, Ph.D., M.Sc., Stephen Rudin, Ph.D., M.Sc., Mary Duffy Fronckowiak, Ph.D., Robert A. Mericle, M.D., and L. Nelson Hopkins, M.D.

Toshiba Stroke Research Center, Department of Neurosurgery, and Departments of Mechanical and Aerospace Engineering and Radiology, State University of New York at Buffalo, Buffalo, New York
\end{abstract}

Successful therapeutic embolization of arteriovenous malformations (AVMs) of the brain with liquid polymers (glues) requires precise knowledge of highly variable AVM structure and flow velocities and transit times of blood through the AVM nidus. The goal of this study was to improve AVM flow measurement and visualization by the substitution of the insoluble Ethiodol (ethiodized oil) contrast agent for the soluble contrast media normally used in angiographic studies.

Before enbucrilate embolization of 24 AVM feeding pedicles in 13 patients, standard contrast medium was superselectively injected into each target pedicle, followed by infusion of $20 \mu \mathrm{l}$ of Ethiodol microdroplets. Transport of contrast material was assessed using high-speed biplane pulsed digital subtraction angiography (DSA) operating at 15 frames per second.

The mean blood flow transit times through AVMs after administration of Ethiodol were found to be approximately half as long as in those measured after injection of soluble contrast materials $(0.22 \pm 0.10$ seconds compared with $0.46 \pm 0.19$ seconds [mean \pm standard deviation]; $\mathrm{p}<0.0001$ ). The discrete Ethiodol microdroplets travel with the core flow, more closely approximating the dynamic behavior of enbucrilate, allowing the AVM structure to be traced with high spatial and temporal resolution. There were no inadvertent vessel occlusions or pulmonary complications related to the use of Ethiodol for DSA.

Because of diffusion and convection, forces that decrease concentration, visualization of the contrast front is reduced, often resulting in deceptively long transit times when soluble contrast materials are used. Overestimation may prove dangerous when planning embolizations. The Ethiodol droplet DSA method provides accurate transit time measurements and precise, detailed, and dynamic AVM visualization. Further development of this method will improve the safety and precision of AVM treatments.

Key Words * brain arteriovenous malformation * enbucrilate embolization * blood velocity * 


\section{Ethiodol droplet tracking * biplane digital subtraction angiography * transit time}

Endovascular embolization has become an accepted adjunct or alternative therapy in the treatment of brain arteriovenous malformations (AVMs). $[4,5,13]$ Over the last few years, many different materials have been tested as occlusive agents in endovascular treatments. These occlusive materials, which include silk thread, polyvinyl alcohol particles, alcohol, detachable coils, and rapidly solidifying liquid adhesive polymers (glues), have been used with varying degrees of success. Only the liquid embolic agents or glues, such as isobutyl cyanoacrylate or enbucrilate, are associated with deep AVM nidus (arteriovenous fistula [AVF] network) penetration and permanent, complete lesion occlusion. However, even with these agents complete, permanent occlusion has been achieved in only 5 to $13 \%$ of cases.[4-6,8,13,32] Moreover, glue embolization carries inherent hazards, such as the risk that the glue will harden too early, in the catheter or proximal feeding artery, or too late, leading to inadvertent occlusion of distal draining veins.

If proximal feeding arteries are occluded without glue penetration into the nidus, revascularization of the AVM through collateral vessels or newly recruited feeding pedicles can occur.[7,11] Inadvertent occlusion of the distal draining veins, on the other hand, can lead to venous hypertension, hemorrhage, or death.[5,19,20] High-flow lesions carry the added risk of possible pulmonary emboli.[4,10,14,15,22,28] To decrease the chance of venous drainage occlusion, a few interventionists have advocated temporary reduction of blood flow, either systemically, by reducing the patient's mean arterial pressure, or locally, by using a calibrated leak system.[5,22] However, the risk of fatal vessel rupture associated with flow arrest is not insignificant and may outweigh the benefits accrued.[22] In short, proper placement of the glue exactly within the AVM nidus, while avoiding the proximal pedicle(s), draining veins, lungs, and the catheter itself, requires extremely good technical skill as well as very careful and precise prediction of embolic agent behavior within the vessel. This, in turn, requires precise knowledge of the complex, extremely variable AVM structure (feeding pedicles, AVF, and draining veins) and velocities and transit times of blood through the AVM nidus. Because none of these parameters has yet been very accurately studied or quantified, the technique of AVM embolization with glue has remained a high-risk procedure, limited by imprecise measurements and understanding, as well as by the technical expertise of the practitioner.

Conventional digital subtraction angiography (DSA), performed at low frame rates and using soluble contrast material, poorly resolves flow rates and transit times, measurements that are needed for precise assessment of glue hardening requirements.[17,25] By substituting Ethiodol, a discrete droplet tracer, for the standard soluble angiographic contrast agent and by implementing high-speed biplane DSA we expect to improve the resolution of the AVM complex and the quantification of hemodynamic parameters, and ultimately to improve the precision, reproducibility, and success of AVM embolizations. Our preliminary results are summarized here.

\section{CLINICAL MATERIAL AND METHODS}

\section{Patient Population}

Angiograms and embolizations were performed in 13 patients, eight females and five males aged 12 to 60 years (mean age 38 years). Before embolization, transit times were calculated in 24 feeding pedicles in a total of 13 AVMs (11 pial, one parenchymal with drainage into a dilated vein of Galen, and one dural AVM) (Table 1). The intravascular use of glues such as Histoacryl and Ethiodol, which is often 
mixed with glue for AVM embolization, is still experimental and has not been approved by the Food and Drug Administration. Therefore, we obtained approval for this study from the institutional review board of the hospital in which all procedures were performed, and informed consent was obtained from each patient or their guardian before treatment.

\begin{tabular}{|c|c|c|c|}
\hline \multicolumn{4}{|c|}{$\begin{array}{c}\text { TABLE } 1 \\
\text { EMEOL RED PED KLES AND CONTRAST MHTER ML TRANSIT TIMES IN } 13 \text { PATIENTS } \\
\text { WITH AVMS }\end{array}$} \\
\hline \multirow[b]{2}{*}{ Pedicle } & \multirow[b]{2}{*}{ AWM L ocation } & \multicolumn{2}{|c|}{ Trarsit Tim es (sec) } \\
\hline & & $\begin{array}{l}\text { Soluble Con- } \\
\text { trast Material }\end{array}$ & Ethiodol \\
\hline 1 & rt frontotem poral & 0.6 & 0.2 \\
\hline 2 & rt frontotem poral & 0.31 & 0.14 \\
\hline 3 & wein of Galen mal form ation & 0.78 & 0.34 \\
\hline 4 & rt tem poral & 0.8 & 0.53 \\
\hline 5 & rt posterior frontal & 0.46 & 0.2 \\
\hline 6 & wein of Galen mal form ation & 0.4 & 0.2 \\
\hline 7 & It dural AVM w/ cortical drainage & 0.53 & 0.34 \\
\hline 8 & wein of Galen mal form ation & 0.41 & 0.28 \\
\hline 9 & wein of Galen mal form ation & 0.34 & 0.21 \\
\hline 10 & rt anterior tem poral & 0.4 & 0.27 \\
\hline 11 & rt tem poroparietal & 0.6 & 0.13 \\
\hline 12 & it tem poroparietal & 0.66 & 0.33 \\
\hline 13 & rt tem poroparietal & 0.93 & 0.2 \\
\hline 14 & rt anterior tem poral & 0.26 & 0.13 \\
\hline 15 & It frontoparietal & 0.33 & 0.2 \\
\hline 16 & It frontoparietal & 0.41 & 0.21 \\
\hline 17 & It frontoperietal & 0.34 & 0.27 \\
\hline 18 & It parietal & 0.4 & 0.2 \\
\hline 19 & It occipitoparietal & & 0.07 \\
\hline 20 & cerebellar & 0.57 & 0.24 \\
\hline 21 & It frontal & 0.60 & 0.33 \\
\hline 22 & It tem poroparietal & 0.20 & 0.13 \\
\hline 23 & It tem poroparietal & 0.26 & 0.13 \\
\hline 24 & It tem poroparietal & 0.4 & 0.13 \\
\hline
\end{tabular}

\section{Superselective Angiography and Transit Time Calculation}

After performing diagnostic angiography in at least two planes (posterior-anterior and lateral), a No. 6 French guide catheter was placed in either the internal carotid artery (ICA) or the vertebral artery for the coaxial introduction of the microcatheter. Before the microcatheter was inserted, the patient was given a bolus of $5000 \mathrm{U}$ of heparin, and the tip of the microcatheter was sprayed with sterile silicone to diminish the risk of inadvertent gluing inside the vessel. The microcatheter was then flow-guided into the AVM's feeding pedicle. In a few cases, a 0.010-in microguidewire was used to achieve satisfactory distal (preferentially wedge) positioning of the microcatheter. Biplane digital road mapping was used for navigation of the microcatheter whenever required.

Standard angiographic studies were performed using a bolus of 0.2 to $1 \mathrm{ml}$ of soluble contrast medium injected through the microcatheter into the feeding pedicle $(3 / 40.5 \mathrm{ml} / \mathrm{second})$. A $240-\mathrm{mg} / \mathrm{ml}$ bolus of organically bound iodine was used in five patients, and a $300-\mathrm{mg} / \mathrm{ml}$ bolus was used in the remaining eight patients. Methohexital sodium (Brevital), an ultrashort-acting barbiturate anesthetic, was administered and provocative testing was then performed (10-mg injection). Ethiodol droplet angiography was performed only after a negative Brevital test and involved the injection of a maximum 
of $20 \mu \mathrm{l}$ of insoluble Ethiodol per feeding artery. The Ethiodol contrast material was injected using a microsyringe attached to the microcatheter through a two-port, Y-shaped valve system, which we designed. This setup, which reduces dead space and flow disturbances, allows the simultaneous and continuous slow infusion of saline solution via the second port, which pushes Ethiodol into the feeding artery.

Ethiodol (ethiodized oil) is a stable organic product formed by iodinating the ethyl esters of the fatty acids in poppyseed oil. At $475 \mathrm{mg}$ of iodine per milliliter, it is $37 \%$ iodine by weight (1.280 specific gravity at 15 šC yields a viscosity of $0.5-1$ poise). Ethiodol has been used previously as a diagnostic contrast agent and, when formed by iodination with iodine-131, as an antineoplastic agent. Our experiments have demonstrated that a small amount of ethiodized oil forms droplets on injection into the arterial bloodstream.[25]

Flow patterns of both soluble and Ethiodol contrast agents were obtained while patients were positioned at the isocenter of a high-speed biplane DSA unit (9-in magnification mode with matrix size $1024 \mathrm{X}$ 1024 for nonselective angiography and 6-in magnification mode with 512 X 512 matrix for high-speed selective angiography). Pulsed angiograms were simultaneously acquired at 15 frames per second (fps) in two optimal projections perpendicular to each other and recorded by two Super VHS analog tape recorders. Travel distances for the contrast media were quantified directly by using the DSA software. Each transit time was calculated from the corresponding videotape by three or more of the authors working in a nonblinded fashion. Details of image postprocessing are described elsewhere.[25] For the initial cases, the Super VHS video frames were digitized using a real-time video digitizer as 512 X 484 matrices, but for the remaining cases, 512 X 512 matrices were directly downloaded to a workstation. Results from these detailed analyses of the Ethiodol contrast imaging patterns are being collected, including precise calculations of blood flow velocities in feeding arteries and draining veins. This work will be presented elsewhere.[26]

The transit time through the AVM is defined as the time interval between initial visualization of injected contrast entering the arterial segment and its subsequent passage into the venous drainage threshold. Transit times for both soluble and Ethiodol contrast agents were calculated in each of the imaging planes. For a given contrast agent, the calculated transit times did not differ significantly between projection planes. In each case, the plane yielding the shortest transit time for soluble contrast was the plane chosen as representative for both types of contrast agents, even if the other projection would have given a slightly shorter Ethiodol transit time. Therefore, differences in transit times between Ethiodol and soluble contrast may, if anything, be slightly underestimated here. Statistical analysis of the transit time differences was performed using a paired t-test.

\section{Embolization Procedures}

The patients received mild sedation prior to the enbucrilate embolization procedure. Enbucrilate was diluted with Ethiodol (ratios ranging from 50:50 to 15:85) for opacification and to vary the hardening time of the glue. The hardening times and injection speeds were chosen to agree with the Ethiodol transit time. After enbucrilate injection (full syringe technique), the microcatheter was rapidly withdrawn with the guide catheter under aspiration to avoid any release of glue from the microcatheter tip. Control angiograms were then obtained, and the introducer sheath was removed. Patients were observed in the neurointensive care unit for 24 to 72 hours.

\section{Sources of Supplies and Equipment}


The Magic 3F/1.5 French infusion microcatheters with olive tip were purchased from Balt, Montmorency, France, and the Quicksilver 10 microguidewire from Medtronic Micro Interventional Systems, Sunnyvale, CA. The Optiray 240 and Isovue $300(240$ and $300 \mathrm{mg} / \mathrm{ml}$ organically bound iodine) were obtained from Mallinckrodt Medical, Inc., St. Louis, MO, and Bracco Diagnostics, Inc., Princeton, NJ, respectively. The Brevital was purchased from Jones Medical Industries, Inc., St. Louis, MO, and Ethiodol from Savage Laboratories, Melville, NY. The CR 700-20 microsyringes were acquired from Hamilton, Reno, NV. The DSA unit (Angiorex Super G model CAS-30B/110A) was provided by Toshiba America Medical Systems, Tustin, CA. The video digitizer equipment (model MV200 with DX digitizer) was obtained from Datacube, Danvers, MA. The computer workstation (Sun Sparcstation 20) was acquired from Sun Microsystems, Mountain View, CA. The enbucrilate embolizing glue (Histoacryl) was obtained from Braun Melsungen AG, Melsungen, Germany.

\section{RESULTS}

We were able to trace the passage of Ethiodol droplets through the AVM with high spatial and temporal resolution, both in the arterial feeding vessels and in the venous system. Droplet blurring was an effective indicator of flow velocity. Even at the high pulse rate used (15 fps), Ethiodol droplets were visible as streaks in high-flow AVM environments such as large draining veins and large feeding arteries (Figs. 1 and 2).

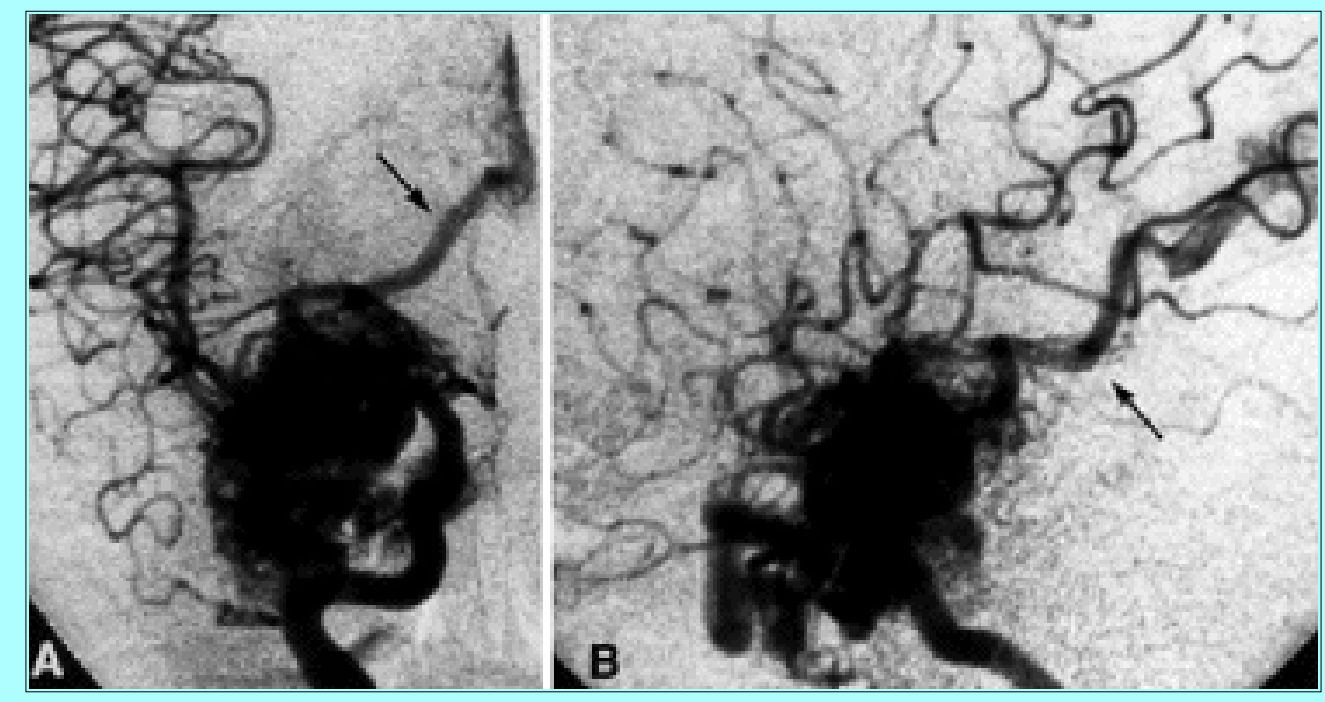

Fig. 1. Diagnostic angiograms of right ICA in posterior-anterior (frontal) (A) and lateral projections (B), showing an anterior temporal AVM supplied by multiple temporal branches of the middle cerebral artery (MCA). The main drainage is into the vein of Rosenthal (arrows). 


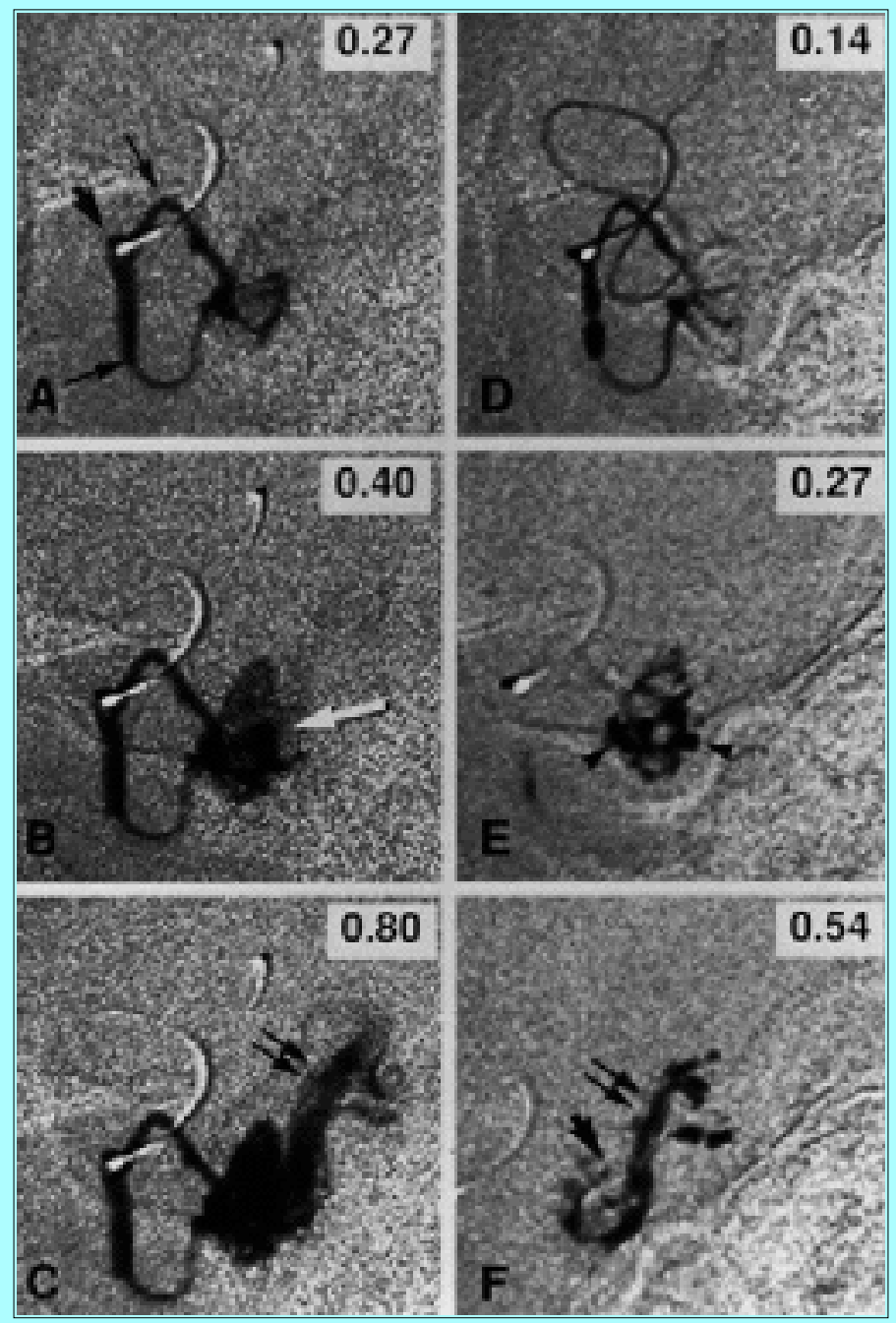

Fig. 2. Same AVM as in Fig. 1. Superselective lateral high-speed angiograms (15 fps) of MCA AVM feeding vessels (Table 1, Pedicle 10). A-C: Angiograms obtained with soluble contrast material showing two main feeding arteries (small arrows), the ill-defined AVM nidus (white arrow), and large draining vein (double arrow). The large arrow in A indicates the position of the microcatheter tip. D-F: Ethiodol droplet angiograms (x-ray pulse width $18 \mathrm{msec}$ ) showing the tortuous distal part of the feeding arteries (arrowheads), the AVFs (large arrow in F), and the draining vein (double arrow). Blurring of droplets inside the vein is caused by high blood flow velocity and large pulse width. The AVM complex is better delineated on Ethiodol-enhanced angiograms, especially the arteriovenous transition. Time in seconds at the top of each image indicates that both feeding arteries, the arteriovenous transition, and the draining vein were opacified earlier after injection of Ethiodol droplets. The transit time for soluble contrast material was calculated as 0.4 seconds and compared with 0.27 seconds for Ethiodol.

However, the droplets were well defined in many small feeding pedicles, indicating low flow. This often occurred before reaching sites of AVFs (transition). Droplet blurring can be reduced by shortening the $\mathrm{x}$-ray pulse width, although equipment limitations prevented us from taking this step earlier in the study. The eventual resolution of these limitations allowed us to reduce the pulse width by approximately a factor of five (from 10-20 down to 2-4) for the last three patients in this study. This alteration markedly reduced blurring, providing much better delineation of the Ethiodol droplets in high-flow conditions (Figs. 3 and 4). 


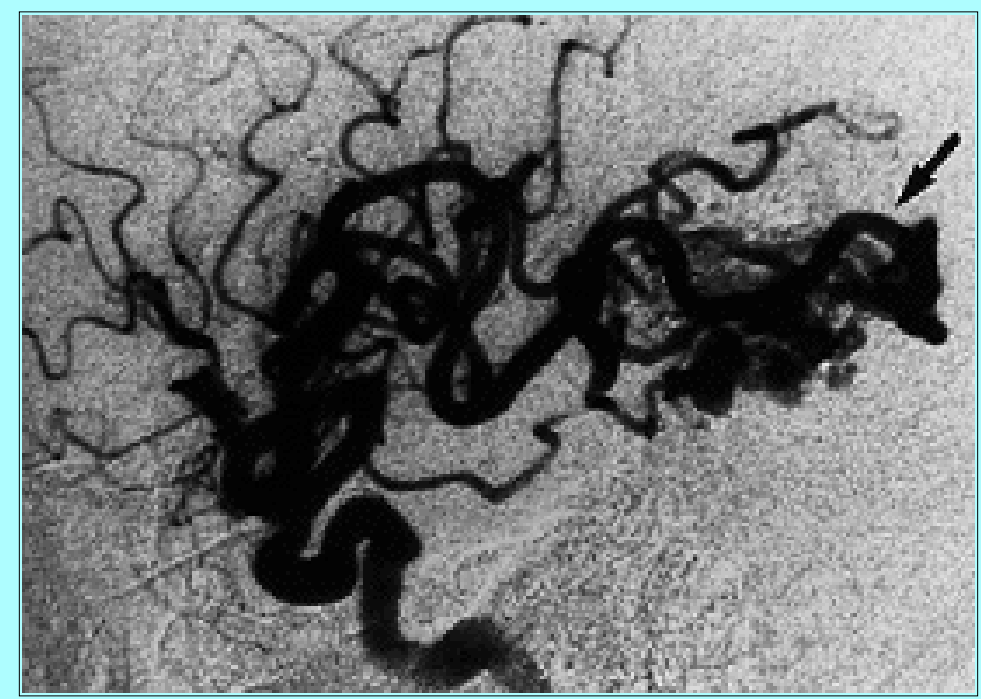

Fig. 3. Diagnostic lateral angiogram of the left ICA showing a temporoparietal AVM supplied mainly by an enlarged parietal branch of the MCA (arrow).

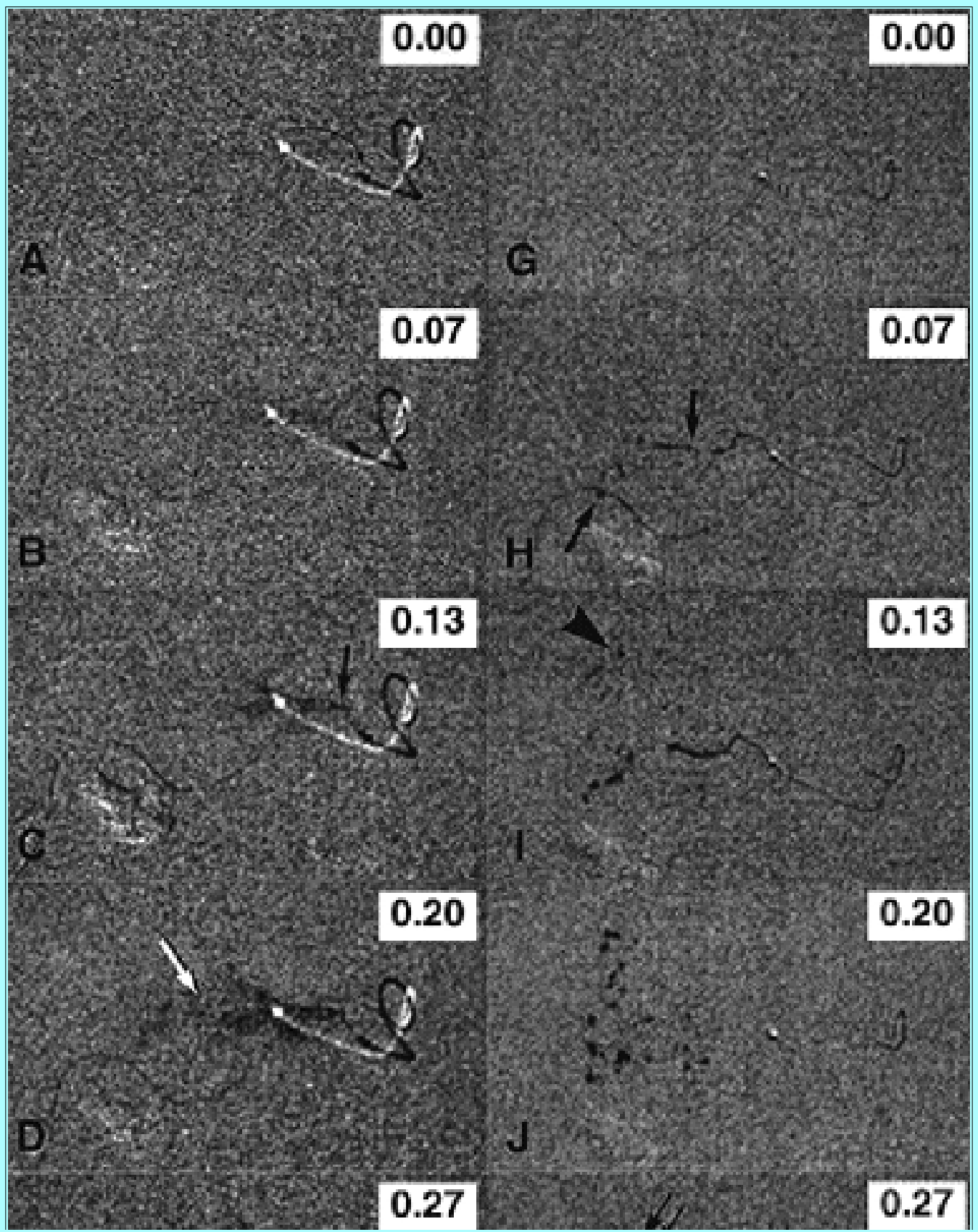




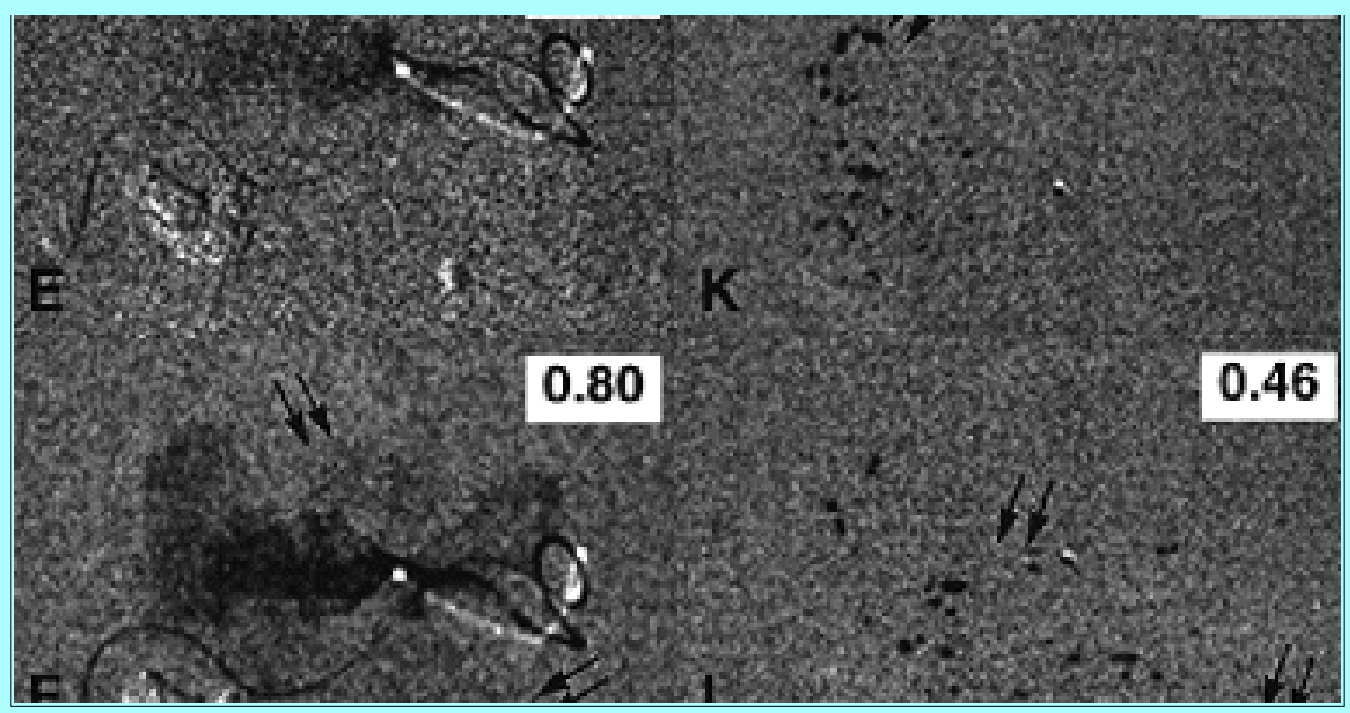

Fig. 4. Same AVM as in Fig. 3. Lateral superselective high-speed (15 fps) angiograms of the parietal high-flow AVM feeding vessel as indicated in Fig. 3 (Table 1, Pedicle 22). A-F: Angiography performed after injection of soluble contrast material showing the large high-flow feeding artery (arrow), the ill-defined arteriovenous transition (white arrow), and large draining veins (double arrows). Note the backlash of the microcatheter tip during forced injection of contrast material needed for opacification (curved arrow in A). G-L: Angiograms showing slow infusion of Ethiodol droplets into the feeding artery (arrows) (x-ray pulse width $2 \mathrm{msec}$ ). Velocity of a well-defined Ethiodol droplet increased immediately after passing through the AVF into the vein (arrowhead). Subsequent droplets are flushed into upper and lower draining veins (double arrows). Time steps in seconds after injection of soluble and insoluble contrast material are indicated at the top of each frame. Transit times for soluble contrast material and Ethiodol were calculated as 0.2 and 0.13 seconds, respectively. Note glue artifact from previous embolization (in lower left of each frame).

In one case, after smaller Ethiodol droplets passed into the draining vein, a group of larger droplets lingered at the arteriovenous transition for several seconds until they were finally carried along by the flow. In summary, details of the AVM complex, including arterial supply and AVF sizes and locations, were more clearly resolved with Ethiodol droplet DSA than with standard, soluble-contrast DSA. Although soluble-contrast DSA provides a complete or near-complete static picture of the AVM angioarchitecture, it cannot be used to detail the overlapping, tangled structural components. In particular, a high-flow lesion frequently appears blurred on soluble-contrast DSA. However, because Ethiodol is a discrete contrast material, it can be tracked with high-frame DSA throughout the AVM complex. This dynamic form of AVM visualization can only be appreciated on videotaped sequences.

\section{Click here to view the video clip that accompanies this article (QuickTime movie).}

The case presented here shows a left frontal AVM in a 45-year-old woman who was admitted to the hospital for a large hematoma. The standard DSA and lateral projection show the AVM nidus, which is composed of midsized multiple arteriovenous shunts. The main supply of the malformation is derived from frontal branches of the middle cerebral artery. The venous phase shows the high arteriovenous shunt as well as the functionally dilated frontal ascending cortical veins and the temporoparietal draining venous system. There is a large venous varix (aneurysm) located dorsally to the AVM nidus. The plain 
$\mathrm{X}$-ray film in lateral projection shows the AVM nidus after previous $\mathbf{N}$-butyl cyanoacrylate (NBCA) embolization. An excellent NBCA case with deep penetration of the AVM fistulas can be seen. The following high-speed lateral angiography shows one of many feeding arteries and the ectatic venous drainage system after superselective catheterization of the AVM; the arrow indicates the catheter tip. The injection phase shows branching of the main arterial feeding vessel into progressively smaller arteries. The injection was performed using up to $1 \mathrm{ml}$ of soluble contrast material (iodine concentration $300 \mathrm{mg} / \mathrm{dl}$ ). Because of the overlap of the arterial and venous phase and diffusion of the contrast material, the exact size and architecture of the arteriovenous transition cannot be shown. However, larger venous draining systems are well depicted. A double contrast injection is performed and the angiographic findings are documented at a high frame rate in lateral projection (30 frames/second). The contrast injection is performed by using soluble contrast material to fill the microcatheter and to infuse the insoluble contrast agent (Ethiodol). A $1 \mathrm{ml}$ syringe is filled with soluble contrast material (iodine concentration $300 \mathrm{mg} / \mathrm{dl}$ ) and the cone of the microsyringe is filled with $20 \mu \mathrm{l}$ of Ethiodol. A slow infusion of the contrast materials is performed. The double contrast DSA shows the droplets being carried away into the venous side, a change in velocity is depicted. This can later be quantified by using any commercial software program.

Transit times calculated after administration of soluble contrast materials were approximately twice as long as those after injection of Ethiodol. Soluble-contrast transit times averaged $0.46 \pm 0.19$ seconds, whereas Ethiodol transit times averaged $0.22 \pm 0.1$ seconds (mean \pm standard deviation). This difference is highly significant, both statistically $(\mathrm{p}<0.0001)$ and clinically for selection of the appropriate enbucrilate-Ethiodol mixture for the embolization.

The scattered datapoints in Fig. 5 plot the individual transit time comparisons of Ethiodol and soluble contrast for each of the 24 AVM feeding vessels studied. All points fall below the dashed line, rather than on it, where they would lie if both contrast agents gave equivalent times. In each and every case, transit times calculated after injection of soluble contrast material are longer than transit times calculated after Ethiodol injection, and some of the individual differences are quite striking. In arterial feeder 13, for instance, the soluble contrast agent yielded a transit time of 0.93 seconds, whereas Ethiodol gave 0.2 seconds; the transit time for the soluble contrast agent was almost five times that of Ethiodol. A least-squares linear regression (solid line) was made to the datapoints in Fig. 5, and the equation for this line is shown in the upper left corner of the plot. As expected, the best fit line indicates that, in general, Ethiodol transit times are approximately half (0.52) as long as soluble-contrast transit times. However, we infer from the square of the correlation coefficient $(0.39)$ that this relationship accounts for little more than one-third of the variation in soluble agent transit time, leaving $60 \%$ of the variation unexplained. 


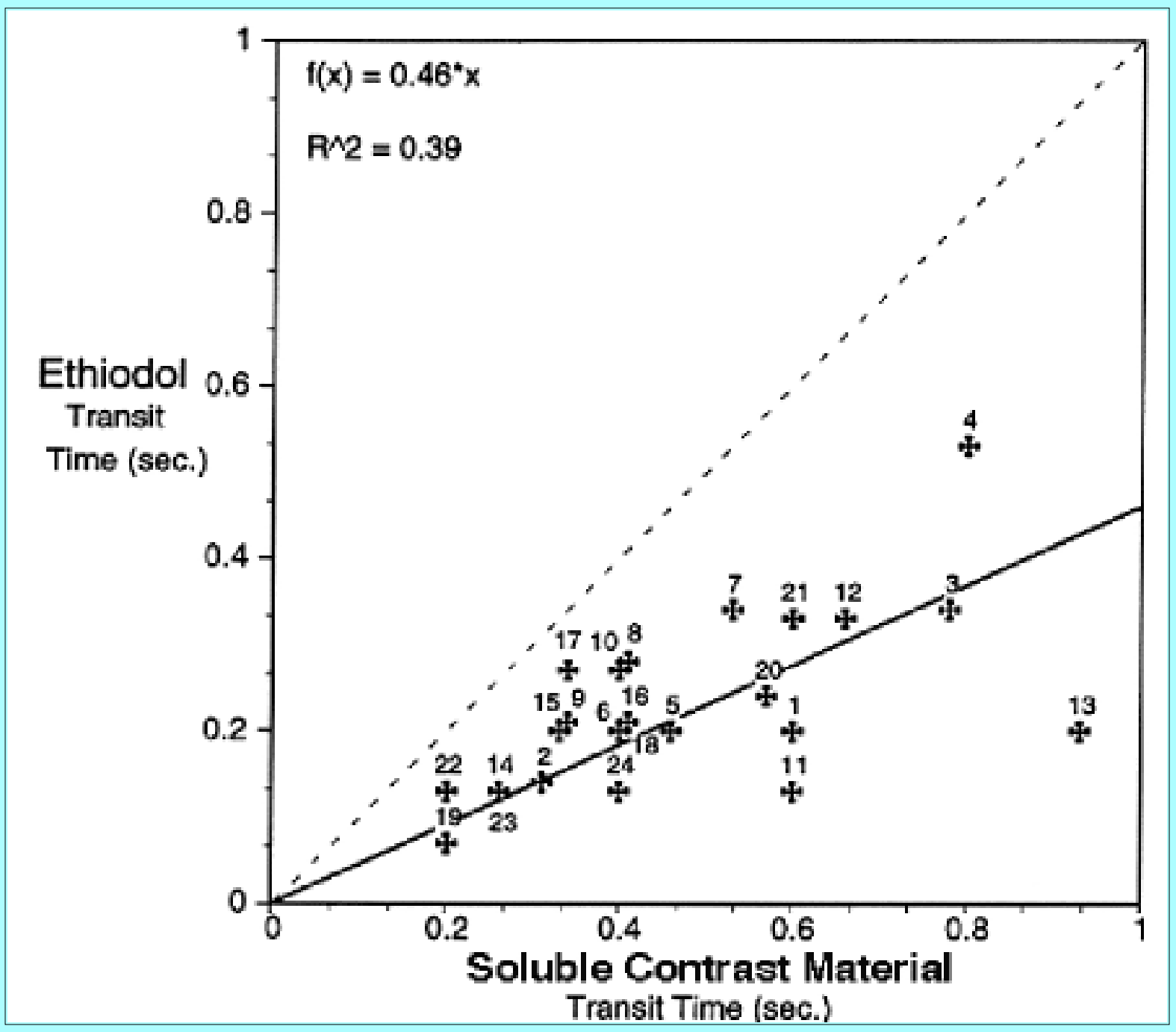

Fig. 5. Graph showing the individual transit time comparisons for Ethiodol compared with soluble contrast material for each of the 24 AVM feeding vessels studied. All points fall below the dashed line, rather than on it, where they would lie if the contrast agents gave equivalent results. The equation for the solid line that was fitted to the datapoints is shown in the upper left corner of the plot. This best fit line indicates that in general Ethiodol transit times are 0.52 times as long as the soluble-contrast transit times. However, the square of the correlation coefficient $(0.39)$ indicates that this relationship is not very rigidly upheld, with little more than one-third of the variation in soluble contrast transit times being directly correlated to variations in Ethiodol transit times. That means that approximately $60 \%$ of the variation in soluble-contrast transit time arises from factors other than the general 2:1 correlation relationship. The transit times for soluble contrast agent averaged $0.46 \pm 0.19$ seconds, whereas those for Ethiodol averaged $0.22 \pm 0.1$ seconds $(\mathrm{p}<0.0001)$.

The discrete Ethiodol droplets provide a benchmark for assessing overestimation of transit time in studies in which soluble-contrast agents were used because they tend to travel with the core flow, away from the slow flow region near the vessel wall, the so-called "boundary layer" where the effects of viscosity come into play. Overestimation of the soluble-contrast transit times is shown in Fig. 6 for the 24 AVM feeding vessels studied here. It can be readily seen that the average value of the overestimation of transit time increases as a direct function of soluble agent transit time, as does the scatter of the individual estimates about this average. A least-squares line was fitted to the datapoints in Fig. 6, and the equation for this line is shown in the upper left corner of the plot. Although the absolute value of the overestimation increases 
as a function of the transit time of soluble contrast material, the relative value is constant throughout. The best fit line indicates that soluble-contrast transit time overestimates that of Ethiodol by a constant relative factor of approximately 2 . The square of the correlation coefficient $(0.74)$ indicates that this correlation is much stronger than that between the respective transit times themselves (0.39). Large values of soluble-contrast agent transit time are much more directly linked to large overestimates of transit time than they are to large values of Ethiodol transit times.

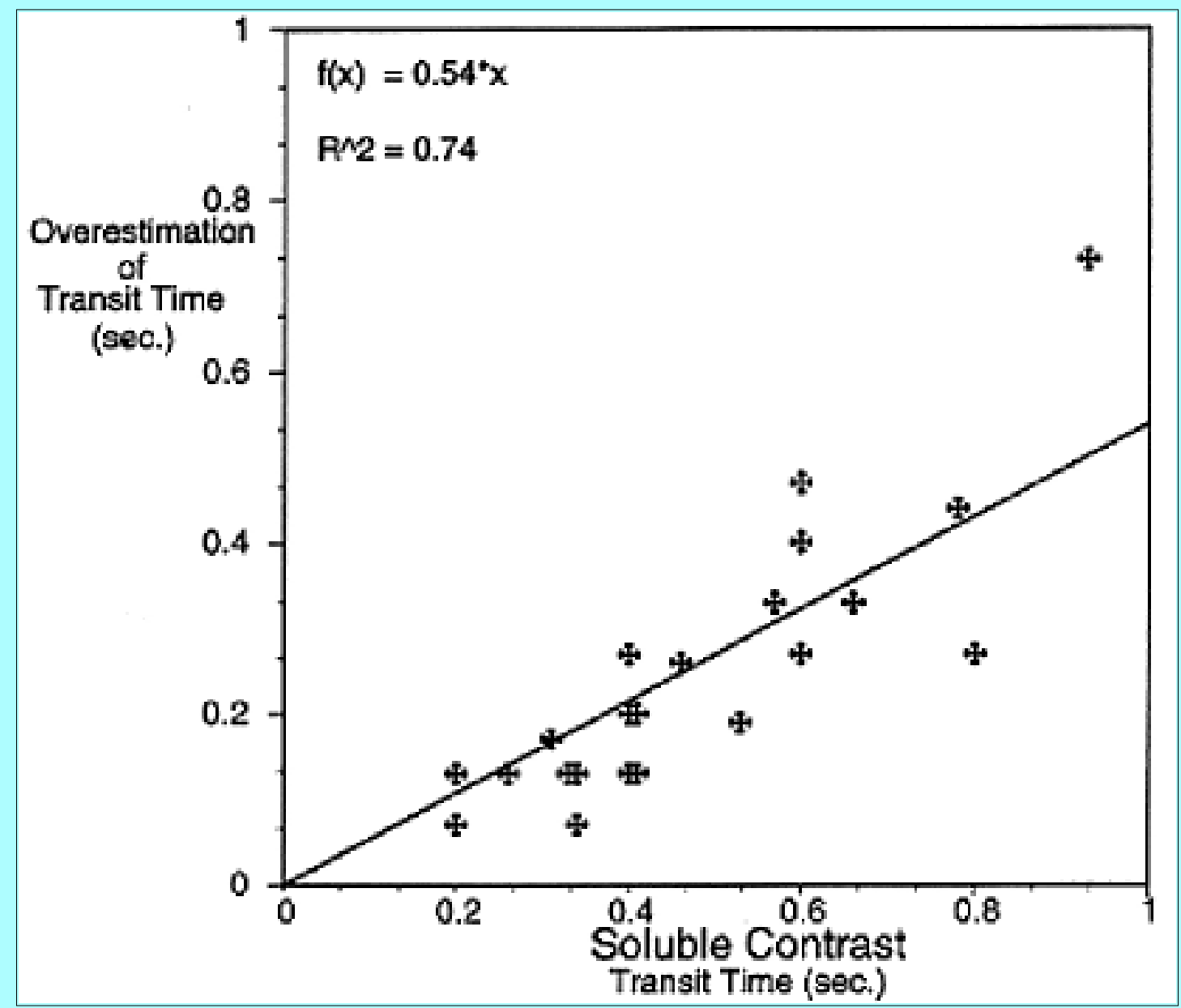

Fig. 6. Graph showing overestimation of transit times by tracing soluble contrast agent for 24 AVM feeding vessels studied using high-speed angiography (15 fps). The average value of the overestimation of transit times increases as a direct function of soluble contrast transit times, as does the scatter of the individual estimates around this average. A least-squares line was fitted to the datapoints. The equation for this line is shown in the upper left corner of the plot. The line indicates that soluble contrast transit times yield overestimates of Ethiodol transit times by a constant relative factor of approximately 2 . The square of the correlation coefficient, 0.74 , indicates that this correlation is much stronger than that between the respective transit times themselves (0.39; see Fig. 5). Large values of soluble contrast transit times are much more directly linked to large overestimates of transit time than they are to large values of Ethiodol transit times.

Intranidal glue casting was safely accomplished in all 24 pedicles, except for two complications, one minor and one transient. In Pedicle 8, a small amount of enbucrilate seen on angiographic studies was washed into a large draining vein with no clinical deterioration. In Pedicle 17, during the third embolization session in a patient with a left frontoparietal AVM, glue was inadvertently deposited into two small draining veins adjacent to the AVM nidus. Twenty-four hours later, the patient developed 
temporary intermittent expressive aphasia that was probably secondary to the focal edema and that resolved after steroid treatment.

No evidence of pulmonary complications from venous embolism related to Ethiodol droplet angiography was found in any of the extensive evaluations that were performed before and after enbucrilate embolization, which included: patient histories and physical examinations, continuous pulse oximetry, analysis of arterial blood gases and, in three patients, chest x-ray films and pulmonary function tests. Moreover, there was no inadvertent occlusion, either transient or permanent, of normal cerebral vessels in connection with Ethiodol droplet DSA.

\section{DISCUSSION}

The Ethiodol droplet DSA method provided detailed, dynamic AVM visualization and accurate AVM transit time measurements. The use of Ethiodol droplets, which approximated the dynamic behavior of enbucrilate, and Ethiodol transit times, which directly reflected core flow, enabled embolizations to be performed more safely and precisely. Ethiodol transit times were significantly shorter than soluble-contrast transit times, differing by approximately a factor of 2 . These observations can be best explained by Hagen-Poiseuille's law[9] for steady laminar flow and the theory of dispersion of soluble matter in steady laminar and turbulent flow developed by Taylor.[29,30] According to Taylor, the concentration gradients of a soluble contrast agent, once injected into steady laminar or turbulent flow, are altered not only by convection but also by diffusion.

\section{Convection and Diffusion}

Suppose one were to inject an insoluble bolus of tracer medium at a given point in the flow field of a pipe in a quantity sufficient to occupy the entire cross section. Further suppose that, at time zero, the interface between this bolus and the surrounding medium is flat. According to Hagen-Poiseuille's law, somewhat later, at time $t$, this flat interface will be stretched into a parabola due to convection, with the vertex or center tip of the flow profile of the bolus moving at twice the average velocity, whereas the sides touching the walls are not moving at all, assuming no-slip boundary conditions (Fig. 7A). However, if one assumes that the insoluble bolus is not injected in sufficient quantity to occupy the entire cross section, or that the bolus is surrounded by a film of moving fluid (as in the case of Ethiodol droplets surrounded by blood), the no-slip boundary condition is lost and a multiphase flow results (Fig. 7B). Insoluble tracer droplets, according to this theory, would be expected to travel at velocities approaching core flow, that is, at twice the average velocity of the moving fluid. 


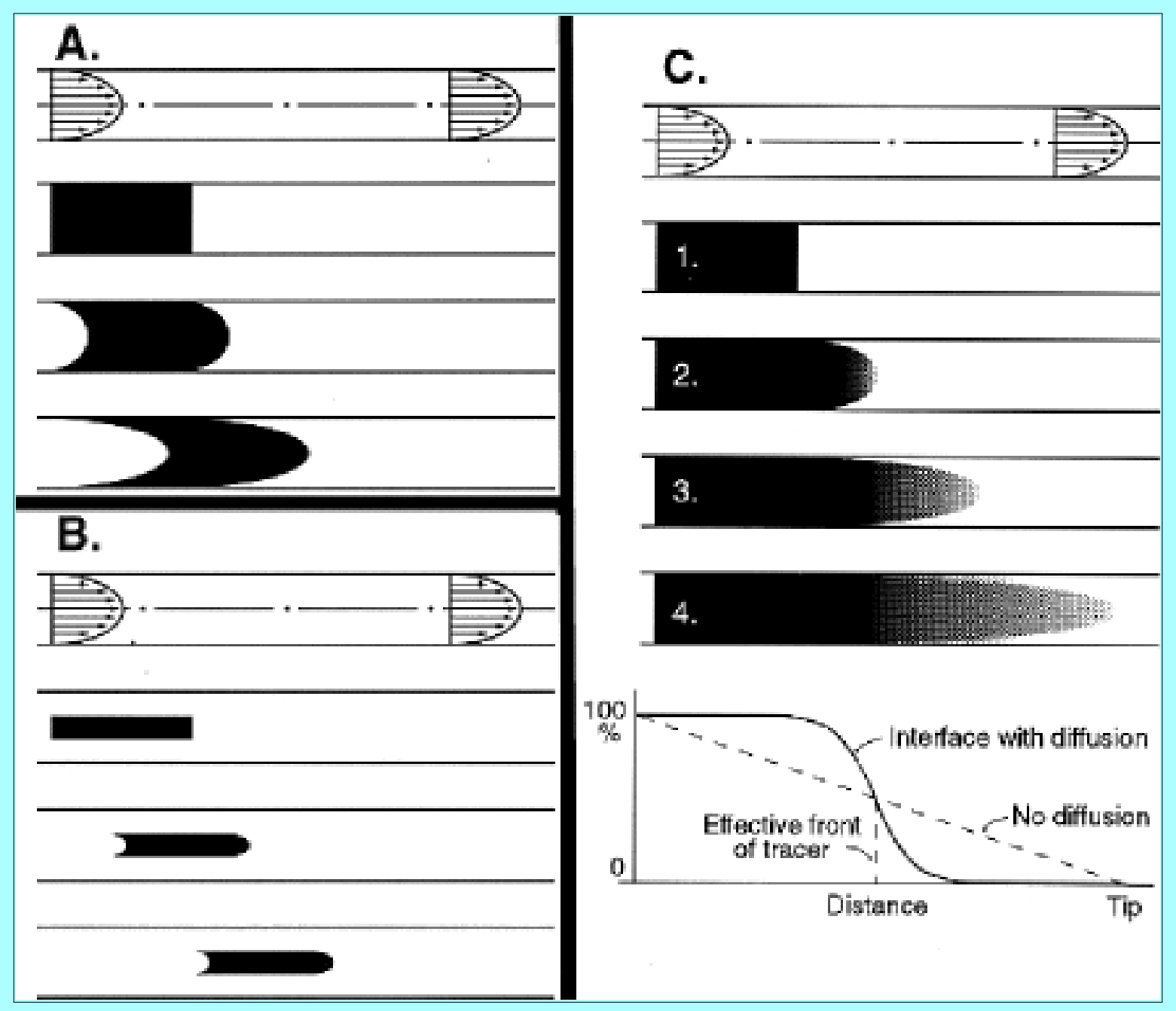

Fig. 7. Schematic drawings illustrating profiles of different tracers injected at time zero $(\mathrm{t}=$ 0 ) into fully developed, steady laminar flow in a pipe. Parabolic velocity profile of the moving fluid is depicted in the upper rows. A: A bolus of insoluble tracer injected at a given point in the flow field of a pipe occupies the entire cross section. At $t=0$, the interface between this bolus and the surrounding medium is flat. Later, at time $t$, this flat interface will be stretched into a parabola. The vertex, or center tip of the bolus, moves at twice the average velocity, whereas the sides touching the walls are not moving at all under no-slip boundary conditions. B: When injected tracer is surrounded by a film of carrying fluid (as in the case of Ethiodol droplets surrounded by blood), the no-slip boundary condition is lost and multiphase flow results. The tracer is expected to travel at velocities approaching core flow, that is, at twice the average velocity of the medium. C: For soluble contrast materials, the concentration profile is modified not only by convection but also by diffusion. Shortly after injection of the soluble material (1), a concentration gradient develops in the longitudinal direction dominated by convection (2). Later (3), a concentration gradient develops in the radial direction, which is dominated by diffusion and becomes more significant with time (4). The solid line in the graph also shows the concentration profile of the material at a later time, as illustrated in (4). The effective front (that is, the front with a sufficient concentration of tracer for visualization) will not be located at the vertex of the velocity profile. But, as the model indicates, the effective front moves at the mean velocity, that is, at half the centerline velocity for laminar flow (or core flow for nonlaminar flow). The concentration shown on the y-axis is averaged over each cross section. The dashed line 
indicates the hypothetical profile in the case of convection alone with no diffusion. (Figure 7C adapted and reprinted with permission from Fung YC, Biomechanics. Motion, Flow, Stress, and Growth. New York: Springer-Verlag, 1990, pp 237-274.)

The theory for soluble media injected in a steady laminar and turbulent flow through a pipe was developed by Taylor in 1953 and 1954.[29,30] The soluble media interface is modified not only by convection as shown by Hagen-Poiseuille's law, but also by diffusion. Shortly after injection of a soluble medium, a concentration gradient develops in the longitudinal direction, dominated by convection (Fig. 7C). Later, a concentration gradient also develops in the radial direction, dominated by diffusion and becoming more significant with time. This radial dispersion significantly modifies the overall concentration profile, or effective front. Specifically, when dealing with soluble contrast materials, the effective front (that is, the front with sufficient concentration of tracer to be visualized) will not be located at the vertex of the parabolic-shaped velocity profile, the core flow region. Rather, Taylor's calculations indicate that the effective front moves at the mean velocity of flow, that is, at one-half the centerline flow velocity for laminar flow or core velocity for turbulent flow.[30] The overall effect is described by Taylor as an apparent diffusion process. The apparent diffusivity is much greater than and inversely correlated with molecular diffusivity.

The observations in our in vivo study appear to follow, generally speaking, the aforementioned theoretical predictions that were based on in vitro observations. Ethiodol droplets, held compactly together by surface tension, traversed the AVM intact with unchanged opaqueness and visibility because they were not subjected to diffusion. They appeared to travel at or near the maximum velocity of the core flow. In AVMs with multiply branching tiny feeding pedicles, Ethiodol droplets fragmented, without marked loss of radiopacity, and were carried into the draining veins. Soluble contrast, on the other hand, which is subject to diffusion, appears to travel at only half the Ethiodol rate, that is, at approximately the mean velocity of flow. Individual variations between the two types of contrast agents ran a much larger gamut, with ratios of soluble-contrast transit times to Ethiodol transit time ranging from a low of 1.3 to a high of 4.7. Fewer factors may have had an impact on the behavior of insoluble contrast materials, causing deviation from theoretical predictions. It is likely that the large variations in soluble-contrast transit times, seen for constant values of Ethiodol times, relate to inherent limitations of soluble contrast materials. For example, consider the variation seen in the following three groupings from Fig. 5: a) Pedicles 2, 11, 14, 22, and 24, where Ethiodol transit time was approximately equal to 0.13 seconds, whereas soluble-contrast transit times ranged from 0.2 to 0.6 seconds; b) Pedicles $1,5,6,9,13,15,16$, and 20, where Ethiodol transit time was approximately equal to 0.2 seconds, whereas soluble transit ranged from 0.33 to 0.93 seconds; and c) Pedicles 3, 7, 12, and 21, where Ethiodol transit time was approximately equal to 0.34 second, whereas soluble-contrast transit times ranged from 0.53 to 0.78 seconds.

There are numerous possible reasons for the deviation of insoluble contrast material flow behavior from that predicted by Taylor's calculations. Simplified assumptions that may not apply to real conditions must be made when studying hemodynamics using models. Figure 7C illustrates the concentration gradients of soluble-contrast medium under conditions of steady laminar flow (Poiseuille's flow), that is, velocity is maintained constant with time. However, pulsatile (non-steady) blood flow amplifies the mixing of soluble contrast materials. Arteriovenous malformations are not fixed lengths of pipes, but rather comprise highly variable and tortuous feeding arteries exhibiting numerous side branches and complex arteriovenous transitions. Conditions are not standardized, optimal, or repetitive; simplified assumptions cannot yield exact predictions, because many other factors come into play. For example, side branches, 
which are not directly opacified, divert contrast agent from the main artery, further diluting the effective soluble-contrast front. The limited contrast-to-noise ratio of the DSA imaging chain, local flow disturbances, and even the technique and force of contrast material injection all affect visualization of the effective contrast front.

Any factor leading to poor opacification of soluble-contrast agents, such as increased diffusivity associated with increased vessel caliber or high AVM flow, will result in falsely long estimates of transit times. Soluble contrast material, by its very definition, is constantly being diluted as it travels through the AVM complex. Any dilution of contrast materials effectively negates, or slows, progression of the visible contrast front, yielding transit times that are overestimates. The longer the transit time of soluble contrast materials, the potentially larger and more variable the overestimate of real transit times, and overestimates are dangerous when planning embolizations, especially in large high-flow AVM feeding vessels. Although angiographic visualization for which current, standard methods are used might be improved by increasing the bolus concentration (the bolus profile), that is, by decreasing the timespan of injection, this, in turn, may boost local flow velocity and flow disturbance, again leading to incorrect transit times. Increased injection force can also lead to whipping, or backlash, and possible displacement of the supple catheter tip (Fig. 4A). Smaller, individual differences in injection rates may explain some of the variation in the transit times for soluble contrast material because three different investigators performed the injections. Operator effect with Ethiodol is estimated to be much less because this contrast material was infused slowly in all cases. To assess the effect of iodine concentration on the effective visibility of soluble-contrast agents, a series of as many as three standard angiograms in each of four patients was obtained while keeping the microcatheter position constant and varying the iodine concentration $(240 \mathrm{mg} / \mathrm{ml}$ compared with $300 \mathrm{mg} / \mathrm{ml})$ in the soluble contrast material. The resultant transit times differed at most by 0.067 seconds, corresponding to a one-frame time step (at $15 \mathrm{fps}$ ). Thus, the iodine concentration in the soluble-contrast medium used in our study did not markedly alter the effective visible front.

With Ethiodol droplet DSA, transit time errors may arise if the ratio of Ethiodol droplet size to blood vessel diameter is too large. In small feeding arteries, this may cause delayed passage of the first insoluble droplet. Delayed passage through an AVF increases resistance to flow, reducing perceived velocity and increasing perceived transit time. Ethiodol transit time calculations should be based on the leading appropriately sized droplet flow. The blurring of Ethiodol droplets that we observed in high-flow situations, such as in draining veins or large feeding arteries, may be reduced by shortening the x-ray pulse. Increased data sampling can be achieved by increasing the DSA frame rate. Together, these methods should increase spatial and temporal resolution, and, ultimately, the accuracy of transit time assessment.

Located far from the aortic root, a cerebral AVM represents a low-impedance system with only very minor fluctuations in blood velocity during the cardiac cycle.[21] These minor fluctuations, which are also observed in small-caliber terminal arteries and arterioles of the systemic circulation,[3] have been estimated to have at most a very negligible effect on the transit time calculations made in this study. Both Ethiodol and soluble contrast materials were injected randomly during the cardiac cycle. If cardiac cycle effects were dominant in this system, any consistent differences between Ethiodol and soluble-contrast transit times would have been washed out by the randomness of the injection process. Instead, we found a very clear pattern. The mean transit times for soluble contrast were two times higher than those for insoluble contrast, and in every instance the individual soluble-contrast transit time was greater than its respective insoluble-contrast transit time. In addition, when repeated injections that were random with 
respect to the cardiac cycle were made in three separate pedicles, the values obtained for transit times of Ethiodol were consistently and repeatedly reproducible for each pedicle.

\section{Embolization Method}

Enbucrilate (Histoacryl)-Ethiodol mixture compositions were chosen based on the past experience and judgment of the endovascular surgeon, because no detailed data concerning the hardening time of these mixtures are available.

The Ethiodol droplets used for angiography have the same approximate viscosity as the enbucrilate-Ethiodol mixtures used for the embolizations, yielding improved simulation of the dynamic behavior of the mixture and improved calculation of transit times. We recently observed a delayed passage of Ethiodol droplets a few millimeters proximal to the AVF in a small feeding artery of an AVM. The glue injection subsequently led to hardening of enbucrilate at that specific location, thus supporting the value of Ethiodol angiography before enbucrilate embolization.

Underestimation of transit time can lead to improper and incomplete AVM nidus glue casting with increased risk for early revascularization, as shown in a recent histological study.[11] On the other hand, overestimation of transit time can result in passage of glue into the cerebral venous drainage or pulmonary systems, with increased risk for cerebral hemorrhage and pulmonary emboli. Use of Ethiodol droplet DSA, with its improved transit times and clearer delineation of fistulas, should enable deeper penetration of the AVM nidus on a routine basis, enhancing the possibility for complete AVM obliteration. Few data are currently available regarding the effects of additives such as Ethiodol or glacial acetic acid on the hardening times of enbucrilate.[22] Glue mixtures are prepared based on limited user experience rather than on precise data; one of the major drawbacks has been an inability to measure precise transit times against which to assess glue behavior. Intranidal glue casting was performed safely here, yet this report was not intended as an outcomes study assessing the success of enbucrilate embolizations using transit time calibration of enbucrilate-Ethiodol mixtures. However, such work is in progress; in an experimental AVM model in swine, we are currently trying to understand the polymerization characteristics of different enbucrilate-Ethiodol mixtures and to quantify and study the variation in hardening times as a function of mixture composition. Better quantification of both AVM transit times and enbucrilate-Ethiodol mixture behaviors could improve the precision with which embolizations are performed, providing the interventionist with the knowledge to make informed choices specific to the clinical situation at hand. This work will be reported elsewhere.

\section{Safety of the Ethiodol Droplet DSA Method}

Ethiodol (ethiodized oil), an ethyl ester of iodinated fatty acid, has been used as a diagnostic contrast agent, mostly in lymphangiography, for more than 30 years. When formed using radioactive iodine-131, Ethiodol has also served as a vehicle for targeted radiation delivery to thyroid tumors. There is a large experience with Ethiodol droplet lymphangiography, because these procedures have been performed in great numbers, often on a routine outpatient basis. The procedure is considered quite safe, although it requires injection of Ethiodol in larger amounts than those used here for vessels feeding brain AVMs, that is, up to $23 \mathrm{ml}$ of Ethiodol in lymphangiography compared with less than $20 \mu \mathrm{l}$ for AVM angiography. Several very large outcome studies have underscored the safety of Ethiodol used as a contrast agent. Hessel, et al.,[12] found no hypotensive reactions in the course of 7641 pedal lymphangiograms, and Koehler,[16] in a much larger study of 32,000 procedures, reported only six cases of hypotensive crisis, a complication rate of $0.02 \%$. These adverse reactions, few as they are, may 
actually represent a response to iodine rather than to Ethiodol itself.[18] In the course of Ethiodol droplet lymphangiography, fine deposits of ethiodized lipid droplets are scattered throughout the pulmonary capillary bed, often showing up as delicate reticular patterns on chest radiography.[1] On occasion, Ethiodol droplets have been associated with an embolization effect, namely decreased pulmonary blood flow and development of acute respiratory distress syndrome after lymphangiography;[27] pulmonary alveolar hemorrhage has also been described as a rare complication of this procedure. Reduction in pulmonary diffusion capacity secondary to the embolization of oil contrast medium may occur in up to $0.4 \%$ of cases after lymphangiography.[12] No specific treatment is required; most patients recover spontaneously in 48 to 72 hours.[1] In the current study, as stated previously, with microliter Ethiodol injections in feeding vessels to 24 brain AVMs, no pulmonary complications were encountered.

Ethiodol emulsions, with droplet sizes less than $3 \mu \mathrm{m}$, have been used in animal studies to assess regional myocardial perfusion, in conjunction with fast computerized tomography.[2] No adverse effects were reported.

It would seem that the sixfold higher than standard DSA frame rates used in this study (15 compared with $2.5 \mathrm{fps}$ ), which are necessitated by shorter AVM transit times with Ethiodol contrast material, would expose both patients and medical personnel to higher radiation risks. However, high frame rates are used in only one small part of the procedure, namely, during selective injection into the AVM feeding vessel. The nonselective part of the diagnostic evaluation is performed at low frame rates ( $2.5 \mathrm{fps})$. Our calculations show that Ethiodol droplet angiography represents at most a $10 \%$ increase in radiation exposure over the course of a single embolization session if the high-speed DSA study is carefully timed to coincide with droplet passage through the field of view. Many adverse radiation effects, including alopecia, erythema, and cataracts, are triggered by a one-time dose above threshold exposure.[31] It is unlikely that a $10 \%$ increase in exposure would change the radiation risk profile substantially for either the patient or the neurointerventionist. Moreover, increased radiation exposure can essentially be avoided altogether by making technical adjustments, for example, reduction of pulse width, if the resulting increase in quantum mottle of the image is acceptable for the procedure. Furthermore, special filters are being developed to be placed between the patient and the x-ray source, further reducing radiation exposure to the patient and investigator. These filters are designed to afford high-quality imaging over a select region of interest, while attenuating x-ray exposure to most of the field of view outside this region. $[23,24]$

\section{CONCLUSIONS}

Blood flow patterns have a substantial effect on arterial disease and on the outcome of therapeutic endovascular procedures to assuage such disease. In this paper, we have proposed an angiographic method in which high-speed pulsed DSA combined with insoluble Ethiodol droplet contrast medium provides improved quantitative measurements of blood flow. This new method could prove to be of great value in both the evaluation of the disease and the development of interventional treatments. The structure of AVMs is inherently complex and variable, yet extremely suitable for quantitative flow assessment by Ethiodol droplet DSA. Our study highlighted the drawbacks of soluble-contrast agents, including sensitivity to factors such as convection-diffusion mixing, which as a consequence yield transit times through AVMs that are highly variable and often overestimated. This overestimation (approximately a factor of 2, range 1.3-5) could prove dangerous when planning therapeutic embolizations. Even at this early stage in its development, high-speed Ethiodol droplet DSA provides much more accurate assessments of core flow, better simulation of the embolic agent in its liquid form, 
much more precise transit time estimates, and greatly improved dynamic visualization of the AVM complex.

\section{Acknowledgments}

We thank Paul Dressel for technical expertise in preparing the illustrations and Debi Zimmer for editorial assistance.

\section{References}

1. Bron KM, Baum S, Abrams HL: Oil embolism in lymphangiography. Incidence, manifestations, and mechanism. Radiology 80:194-202, 1963

2. Canty JM Jr, Judd RM, Brody AS, et al: First-pass entry of nonionic contrast agent into the myocardial extravascular space. Effects on radiographic estimates of transit time and blood volume. Circulation 84:2071-2078, 1991

3. Chandran KB: Cardiovascular Biomechanics. New York: New York University Press, 1992, pp $22-33$

4. Coard K, Silver MD, Perkins G, et al: Isobutyl-2-cyanoacrylate pulmonary emboli associated with occlusive embolotherapy of cerebral arteriovenous malformations. Histopathology 8:917-926, 1984

5. Debrun GM, Aletich V, Ausman JI, et al: Embolization of the nidus of brain arteriovenous malformations with n-butyl cyanoacrylate. Neurosurgery 40:112-121, 1997

6. Deruty R, Pelissou-Guyotat I, Mottolese C, et al: The combined management of cerebral arteriovenous malformations. Experience with 100 cases and review of the literature. Acta Neurochir 123:101-112, 1993

7. Fournier D, TerBrugge K, Rodesch G, et al: Revascularization of brain arteriovenous malformations after embolization with bucrylate. Neuroradiology 32:497-501, 1990

8. Fournier D, TerBrugge KG, Willinsky R, et al: Endovascular treatment of intracerebral arteriovenous malformations: experience in 49 cases. J Neurosurg 75:228-233, 1991

9. Fung YC: Biomechanics: Motion, Flow, Stress, and Growth. New York: Springer-Verlag, 1990, pp $237-274$

10. Goldman ML, Philip PK, Sarrafizadeh MS, et al: Transcatheter embolization with bucrylate (in 100 patients). Radiographics 2:340-375, 1982

11. Gruber A, Mazal PR, Bavinzski G, et al: Repermeation of partially embolized cerebral arteriovenous malformations: a clinical, radiologic, and histologic study. AJNR 17:1323-1331, 1996

12. Hessel SJ, Adams DF, Abrams HL: Complications of angiography. Radiology 138:273-281, 1981

13. Hurst RW, Berenstein A, Kupersmith MJ, et al: Deep central arteriovenous malformations of the brain: the role of endovascular treatment. J Neurosurg 82:190-195, 1995

14. Hutton L, Fox AJ, Viñuela F, et al: Iatrogenic pulmonary embolism due to embolization of 
cerebrovascular lesions. AJNR 5:665, 1984 (Abstract)

15. Kjellin IB, Boechat MI, Viñuela F, et al: Pulmonary emboli after embolization of cerebral arteriovenous malformations in children. Radiology 201 (Suppl):237, 1996 (Abstract)

16. Koehler PR: Complications of lymphography. Lymphology 1:116-120, 1968

17. Lieber BB, Rudin S, Wakhloo AK, et al: Fluoroscopic particle tracking in a model aneurysm using traceable droplets. Adv Bioeng 32:457-458, 1996

18. Lossef SV, Barth KH: Severe delayed hypotensive reaction after Ethiodol lymphangiography despite premedication. AJR 161:417-418, 1993

19. Lundqvist C, Wikholm G, Svendsen P: Embolization of cerebral arteriovenous malformations: part II--aspects of complications and late outcome. Neurosurgery 39:460-469, 1996

20. Massoud TF, Duckwiler GR, Viñuela F, et al: Acute subdural hemorrhage complicating embolization of a cerebral arteriovenous malformation. AJNR 16:852-856, 1995

21. Nornes H, Grip A: Hemodynamic aspects of cerebral arteriovenous malformations. J Neurosurg 53:456-464, 1980

22. Pelz DM, Lownie SP, Fox AJ, et al: Symptomatic pulmonary complications from liquid acrylate embolization of brain arteriovenous malformations. AJNR 16:19-26, 1995

23. Rudin S, Bednarek DR: Region of interest fluoroscopy. Med Phys 19:1183-1189, 1992

24. Rudin S, Guterman LR, Granger WE, et al: Application of region-of-interest imaging techniques to neurointerventional radiology. Radiology 199:870-873, 1996

25. Rudin S, Lieber BB, Wakhloo AK, et al: Quantitative flow velocity measurements in vessels, aneurysms, and arteriovenous malformations (AVMs) using droplet path tracing with a biplane pulsed fluoroscopy system. SPIE Proc Med Imaging 3033:268-279, 1997

26. Rudin S, Wakhloo AK, Lieber BB, et al: Droplet tracking using biplane digital angiography for cerebral arteriovenous malformation blood flow velocity and vessel morphology determinations.

Radiology 205 (Suppl):354, 1997 (Abstract)

27. Silvestri RC, Huseby JS, Rughani I, et al: Respiratory distress syndrome from lymphangiography contrast medium. Am Rev Respir Dis 122:543-549, 1980

28. Takasugi JE, Shaw C: Inadvertent bucrylate pulmonary embolization: a case report. J Thorac Imaging 4(4):71-73, 1989

29. Taylor G: The dispersion of matter in turbulent flow through a pipe. Proc R Soc (A) 223:446-468, 1954

30. Taylor G: Dispersion of soluble matter in solvent flowing slowly through a tube. Proc R Soc (A) 219:186-203, 1953

31. Wagner LK: Biologic effects of high x-ray doses, in Balter S, Shope TB (eds): Syllabus: A Categorical Course in Physics: Physical and Technical Aspects of Angiography and Interventional 
Radiology. Oak Brook, IL: Radiological Society of North America, 1995, pp 167-170

32. Wikholm G, Lundqvist C, Svendsen P: Embolization of cerebral arteriovenous malformations: part I-- technique, morphology, and complications. Neurosurgery 39:448-459, 1996

Manuscript received November 4, 1997.

Accepted in final form April 10, 1998.

This study was supported by Biomedical Engineering Research Grant No. 95-0028 from the Whitaker Foundation, the Ralph Hochstetter Medical Research Fund in Honor of Dr. Henry C. and Berta H. Buswell (A.K.W.), and Grant No. R01NS31883 from the National Institute of Neurological Diseases and Stroke, National Institutes of Health (S.R.).

Address reprint requests to: Ajay K. Wakhloo, M.D., Ph.D., c/o Editorial Office, Department of Neurosurgery, State University of New York at Buffalo, 3 Gates Circle, Buffalo, New York 14209-1194. email: awakhloo@acsu.buffalo.edu. 


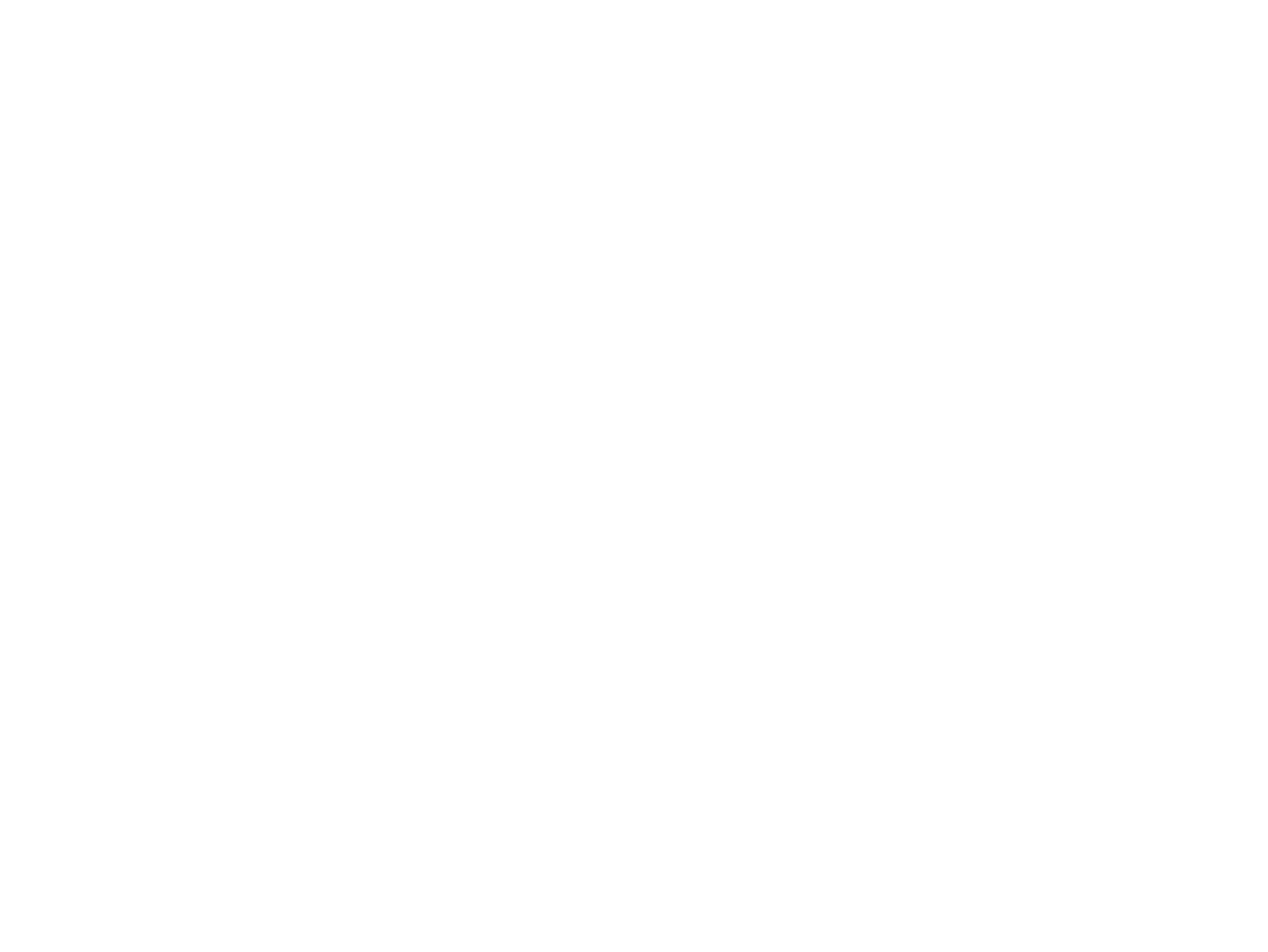

Unauthenticated | Downloaded 04/26/23 02:28 PM UTC 